\title{
The effect of agmatine on trichothecene type B and zearalenone production in Fusarium graminearum, $F$. culmorum and F. poae
}

Matias Pasquali, Emmanuelle Cocco, Cedric Guignard, Lucien Hoffmann

Agmatine and other putrescines are known strong inducers of deoxynivalenol (DON) production in Fusarium graminearum. Other important cereal species produce DON and/or other trichothecene type B toxins (3 acetylated DON, 15 Acetylated DON, Fusarenon-X, Nivalenol) such as F. culmorum and $F$. poae. In order to verify if the mechanism of regulation of trichothecenes type $B$ induction by agmatine is shared by different species of Fusarium, we tested the hypothesis on 19 strains belonging to 3 Fusarium species ( $F$. graminearum, F. culmorum, F. poae) with diverse genetic chemotypes (3ADON, 15ADON, NIV) by measuring trichothecene $B$ toxins such as DON, NIV, Fusarenon-X, 3ADON and 15ADON. Moreover we also tested whether other toxins like zearalenone were also boosted by agmatine. The trichothecene type B boosting effect was observed in the majority of strains used (13 over 19) in all the three species. Representative strains from all the three genetic chemotypes were able to boost toxin production after agmatine treatment. We identified strains not responding to the agmatine stimulus which may contribute to deciphering the regulatory mechanisms that link toxin production to agmatine (and more in general polyamines). 
1 The effect of agmatine on trichothecene type B and zearalenone production in Fusarium

2 graminearum, F. culmorum and $F$. poae.

3

4 Matias Pasquali*, Emmanuelle Cocco, Cedric Guignard and Lucien Hoffmann

5

6 Luxembourg Institute of Science and Technology (LIST), "Environmental Research and

7 Innovation” Department (ERIN), 41, rue du Brill, L-4422 Belvaux, Luxembourg;

8 *Author to whom correspondence should be addressed; E-Mail: matias.pasquali@list.lu;

$9 \quad$ Tel.: +3524702 436 - 458; Fax: +352 275885 .

11 Abstract: Agmatine and other putrescines are known for being strong inducers of 12 deoxynivalenol (DON) production in Fusarium graminearum. Other important species 13 produce DON and/or other trichothecene type B toxins (3 acetylated DON, 15 Acetylated DON, Fusarenon-X, Nivalenol), such as F. culmorum and F. poae. In order to verify

15 whether the mechanism of the regulation of trichothecene type B induction by agmatine is 16 shared by different species of Fusarium, we tested the hypothesis on 19 strains belonging 17 to 3 Fusarium species (F. graminearum, F. culmorum, F. poae) with diverse genetic 18 chemotypes (3ADON, 15ADON, NIV) by measuring trichothecene B toxins such as DON, 19 NIV, Fusarenon-X, 3ADON and 15ADON. Moreover, we tested whether other toxins like 20 zearalenone were also boosted by agmatine. The trichothecene type B boosting effect was 21 observed in the majority of strains (13 out of 19) in all the three species. Representative 
22 strains from all three genetic chemotypes were able to boost toxin production after

23 agmatine treatment. We identified the non-responding strains to the agmatine stimulus,

24 which may contribute to deciphering the regulatory mechanisms that link toxin production

25 to agmatine (and, more generally, polyamines).

\section{Introduction}

Mycotoxin regulation mechanisms leading to accumulation of toxins in the plant, and consequently in grains used for human and animal consumption, are still partially unknown. Nutrients and specific molecules are supposed to play a key inducing role in activating toxin pathways in planta. Fusarium graminearum Schwabe [teleomorph Gibberella zeae (Schwein.) Petch], Fusarium culmorum (WG Smith) Sacc. and Fusarium poae (Peck) Wollenw. are the main species associated with trichothecene type B (TB) production in Fusarium Head Blight (FHB) in wheat in different agricultural areas in the world. The most important Fusarium TB are deoxynivalenol (DON), the amount of which in food and feed is controlled in many countries, 3acetylated DON (3ADON), 15-acetylated DON (15ADON), Nivalenol (NIV) and Fusarenon X (FUSX). F. graminearum and F. culmorum can also produce Zearalenone (ZEA), a toxin which is legislatively regulated in food and feed due to its estrogenic effect on humans and animals.

41 Each Fusarium strain is able to produce some of these toxins depending on the set of genes

42 present in the genome (Proctor et al., 2009) and the triggering factors leading to toxin 43 production. Because toxigenic risk in food depends on multiple factors including the type of 
44 fungal population colonizing the plant (and its toxigenic potential), much effort has been devoted 45 to studying the epidemiology of different chemotypes worldwide (Pasquali and Migheli, 2014), 46 using genetic means to discriminate populations that are more or less toxigenic (von der Ohe et 47 al., 2011). Chemical determination of chemotypes is still an important approach to confirm genetic analysis based on gene polymorphisms (Desjardins, 2008); therefore liquid media able to stimulate toxin production have been widely employed for determining the toxin potential of isolates.

Mechanisms of toxin induction in F. graminearum include sugar types (Jiao et al., 2008), pH effects (Gardiner et al., 2009; Merhej et al., 2010), inorganic compounds (Tsuyuki et al., 2011; Pinson-Gadais et al., 2008), oxidative stress (Ponts et al., 2007), fungicides (Magan et al. 2008), light (Kim et al., 2014), and water activity levels (Llorens et al., 2004; Schmidt-Heydt et al., 55 2011) and have also been linked to chemotype diversity (Ponts et al., 2009). F. graminearum boosts toxin production when grown in a medium with agmatine and other putrescines to levels that are comparable to the high contaminations observed in planta (Gardiner et al., 2009b). Hypotheses on the role of polyamines in planta as a cue for the production of trichothecene mycotoxins by $F$. graminearum during the FHB disease have been formulated (Gardiner et al., 2010) and the use of inhibitors of polyamine transport in the fungal cells have been proposed as a novel approach to limit toxin contamination in grains (Crespo-Sempere et al., 2015). Studying the effect of agmatine on other species can elucidate whether this mechanism is also effective in other Fusarium species that are often found to coexist in agricultural settings (Giraud et al., 2010), directly contributing to toxin accumulation (Beyer et al., 2014). Because the colonization of the plant by the fungus and the resulting production of toxins are the outcomes of the interaction of the environment, the fungus and the plant, here we focused on how fungal 
67 diversity is affected by a putative plant-derived inducer of toxin production. We therefore tested

68 the level of toxin induction in relation to fungal diversity in 3 Fusarium species when confronted

69 with 2 standardized media which are mild and strong inducers of toxin synthesis.

70 The aims of the work were therefore to evaluate the toxigenic potential in vitro of a set of

71 isolates and to analyse the effect of agmatine as toxin-inducing compounds across species and

72 chemotypes.

\section{Material and methods}

75

76

77

\subsection{Isolates and growth conditions}

Isolates used in this study are listed in table 1. As determined in our laboratory, they have a different geographic origin and belong to different genetic chemotypes (Pasquali et al., 2011).

Strains are conserved in the Luxembourg Microbial Culture Collection accessible at

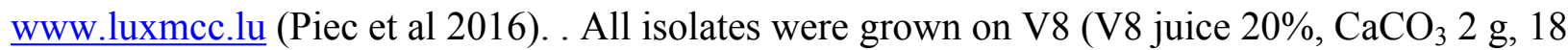
g agar, $\mathrm{H}_{2} \mathrm{O}$ to $1 \mathrm{~L}$ ) plates for 4 days at $20^{\circ} \mathrm{C}$ in the dark. The growing edge of the mycelium was divided into $2 \mathrm{~mm}$ squares and one square each was used to inoculate $10 \mathrm{ml}$ media in sterile glass tubes. The assay was carried out with three completely independent biological replicates.

In order to analyse toxin induction, 2 media were used and compared. The first medium (J), from Jiao et al., 2008 (containing $1 \mathrm{~g} \mathrm{~K}_{2} \mathrm{HPO}_{4}, 0.5 \mathrm{~g} \mathrm{KCl}, 0.5 \mathrm{~g} \mathrm{MgSO} 4 \cdot 7 \mathrm{H}_{2} \mathrm{O}, 2 \mathrm{~g}$ L-glutamic acid, $10 \mathrm{mg} \mathrm{FeSO} \cdot 7 \mathrm{H}_{2} \mathrm{O}, 10 \mathrm{~g}$ sucrose in a litre of solution) was compared to a medium (G) from Gardiner et al., (2009b), containing (30 g sucrose, $1.15 \mathrm{~g}$ Agmatine, $1 \mathrm{~g} \mathrm{KH}_{2} \mathrm{PO}_{4}, 0.5 \mathrm{~g}$ $\mathrm{MgSO}_{4} \cdot 7 \mathrm{H}_{2} \mathrm{O}, 0.5 \mathrm{~g} \mathrm{KCl}, 10 \mathrm{mg} \mathrm{FeSO} \cdot 7 \mathrm{H}_{2} \mathrm{O}$ in a litre solution). The two media have 
88 approximately the same $\mathrm{C} / \mathrm{N}$ ratio, which is known to play a role in toxin regulation (Hestbjerg et

89 al., 2002) but differ for the source of nitrogen and the amount of carbon source. Tubes were

90 shaken at $180 \mathrm{rpm}$ in the dark for $10 \mathrm{~d}$. Fungal biomass was then filtered and weighted with a

91 precision balance after drying using a freeze drier for $48 \mathrm{~h}$, and the remaining media were kept

92 for further toxin extraction.

93

94

95

96

97

98

99

100

101

102

103

104

105

106

107

108

109

\subsection{Chemical analysis}

The medium was filtered through a $0.2 \mu \mathrm{m}$ GHP membrane filter (PAL, MI, USA) and diluted in methanol (extract/methanol, $9 / 1, \mathrm{~V} / \mathrm{V})$ in order to be in the appropriate solvent ratio for chromatographic analysis. The quantification of all mycotoxins except 3-ADON and 15-ADON was performed by LC coupled to tandem mass spectrometry (LC-MS/MS, Dionex Ultimate 3000, AB/Sciex API 3200, Foster City, CA, USA) in multiple reaction monitoring (MRM) in positive/negative switching mode. The LC analytical column was an Agilent Zorbax Eclipse Plus $\mathrm{C}_{18}(2.1 \mathrm{X} 150 \mathrm{~mm}, 3.5 \mu \mathrm{m})$ with a mobile phase consisting of methanol and water containing 2.5 $\mathrm{mM}$ of ammonium acetate in a linear gradient. The specific analysis of 3-ADON and 15-ADON was achieved using a second LC-MS/MS method on an Agilent 1260 LC coupled to an AB/Sciex 4500 QTrap mass spectrometer. The column was an Agilent Poroshell 120 EC-C 18 $(2.1 \times 150 \mathrm{~mm}, 2.7 \mu \mathrm{m})$ and the eluents were the same as for the first method. All mycotoxins were quantified by external calibration based on pure standards (Biopure, Tulln, AT and SigmaAldrich, Schnelldorf, DE). The detection and quantification limits were $1.5 \mathrm{ng} / \mathrm{ml}$ of liquid culture for all toxins. The analyses were done in two technical replicates and the average value was considered for each biological replicate.

\subsection{Statistical analysis}


110 All data were analysed using PASW version 19 and SigmaPlot version 12.5. Overall TB

111 production was calculated summing up all trichothecenes type B measured (DON, 3ADON,

112 15ADON, NIV, FUS-X). Mass-corrected toxin concentrations were calculated and used for

113 determining the effect of nitrogen source on toxin boosting. The Mann-Whitney Rank Sum test

114 was used to verify the effect of the medium on masses and the effect of nitrogen source in the

115 medium on mass-corrected summed trichothecene type B values. The Kruskal-Wallis One-Way

116 Analysis of Variance on Ranks was used to verify the effect of the medium on the average ratio

117 of TB production in the two media for each strain classified either as species or as chemotypes.

118 Significant differences are considered when $\mathrm{p}<0.05$.

\section{Results and Discussion}

122 Growth (dry mass) and toxin production using a multimethod assay including DON, 3ADON, 15ADON, NIV, FUS-X, ZEA, T2, HT2 were measured on a set of 19 strains (table 1) when grown in the two media. Mycelium growth (estimated as dry mass) was influenced by the medium increasing, as expected, in the high containing saccharose medium $(\mathrm{p}<0.001)$. The mass

126 of the strains in glutamic acid medium ranged from $3.4 \mathrm{mg}$ to $19.6 \mathrm{mg}$ while in the

127 agmatine/saccharose-rich medium, masses ranged from 8.9 to $36.1 \mathrm{mg}$. Growth results are 128 comparable with results obtained by Jiao et al., (2008) for the medium with glutamic acid. We 129 observed that FG and FC had a similar range of growth in each medium while FP differed 130 significantly in growth compared to the other two species $(p>0.05)$. No effect of the chemotype $131(\mathrm{p}=0.328)$ could be identified. In all three species there was nonetheless a significant growth 
132 effect caused by the medium being the agmatine/high saccharose medium, a booster of mass

133 growth ( $\mathrm{p}>0.001$ for all the three comparisons, figure 1$)$. The overall increase in dry mass caused

134 by the agmatine medium can be attributed to the higher amount of saccharose in the medium,

135 with carbon being the main constituent of fungal biomass (Newell et al., 1982). To evaluate toxin

136 production, we therefore normalized the toxin produced by the dry mass per ml.

137 The two sources of nitrogen had an overall significant effect on trichothecene type B mass-

138 corrected production across the three species $(p=0.003)$. The agmatine medium induced higher

139 TB production in 13 out of 19 strains. Six isolates produced average TB toxins in $\mathrm{ng} / \mathrm{ml}$

140 corrected by their mass in $\mathrm{mg}$ above $150 \mathrm{ng} / \mathrm{ml} / \mathrm{mg}($ F. culmorum 233, 13-01; F. graminearum

141734,37099 and MUCL42825 and F. poae 80). All these isolates increased their production in the

142 medium containing agmatine with a boosting factor ranging between 192X of 13-01 to $15 \mathrm{X}$ of

143 isolate 80. Four isolates (01-02, 557. MUCL15500, UMW00706) demonstrated the opposite

144 behaviour when grown in the medium containing agmatine (figure 2).

145 We could also confirm that strains with a determined genetic chemotype can also produce a

146 minor amount of the other acetylated and non-acetylated trichothecenes (De Kuppler et al.,

147 2011). Nonetheless, there was a good correspondence between the genetic chemotype and the

148 major toxin type produced (Supplementary table 1).

149 By analysing the average ratio of toxin production in the isolates according to their species or

150 their chemotype, we could not detect a clear effect of any of the two categories. In fact, the

151 response of toxin production to the two media was not significantly affected by the species

$152(\mathrm{p}=0.552)$ nor by the chemotype $(\mathrm{P}=0.578)$. A previous hypothesis suggesting differences at the

153 chemotype level on toxin regulation (Ponts et al., 2009) may have been biased by the limited

154 number of strains used. 
155 ZEA production, in the only consistent producer of the lot (F. culmorum 01-02), doubled in the

156 agmatine medium suggesting that the mechanism boosting TB production also potentially affects

157 ZEA production. This would imply that the agmatine regulation of ZEA and of the trichothecene

158 cluster is common, despite gene expression data from the available microarray studies not

159 showing consistent concordant patterns (Sieber et al., 2014). A larger set of isolates producing

160 ZEA is nonetheless needed to verify this hypothesis.

161 In our analysis, we also included two food and feed monitored trichothecene type A (TA) toxins

162 but no isolates of $F$. poae produced any detectable amount of T2 and HT-2. As the biosynthesis

163 of TAs follows a similar pathway (Kimura et al., 2007), we can expect similar effects due to

164 agmatine on TA. However, we could not confirm this with the isolates used in our study.

\section{4. Conclusions}

166 The effect of 2 different media containing agmatine or glutamic acid on toxin induction and

167 growth in a total of nineteen strains belonging to the $F$. graminearum $(n=7), F$. $\operatorname{culmorum}(n=8)$

168 and $F$. poae $(n=4)$ species was evaluated. With our species-comparative study, we confirmed

169 that on average the mechanism of triggering toxin production by agmatine is confirmed in the 3

170 species but that the mechanism is also significantly influenced by strain diversity (Pasquali et al.,

171 2015). The identification of isolates that do not respond to agmatine may help decipher the

172 pathways leading to specific agmatine regulation in the fungus. Despite these data being obtained

173 by culturing the fungus in vitro, they can contribute to explaining the partial diversity of toxin

174 contamination observed in planta: indeed, on average, different cultivars accumulate different

175 levels of DON (Ji et al., 2015). Hypotheses on the effect of the plant oxidative status

176 (Waśkiewicz et al., 2014) and of polyamine concentration on toxin synthesis in planta have been

177 formulated (Gardiner et al., 2010). Here we showed that strain diversity can also account for a 
178 factor 1000X in the level of toxin accumulation. Exploring the diversity of interactions between

179 strains and toxin triggering compounds such as polyamines (Valdes Santiago et al., 2012) is

180 fundamental to identifying general inhibitors of TB accumulation in the plant.

\section{Acknowledgments}

183 We thank Laurence Joly for her technical assistance with toxin measures and Marine Pallez for

184 her support in fungal collection maintenance. Lindsey Auguin is acknowledged for the English 185 revision of the manuscript.

186

187

188

189

190

191

192

193

194

195

196

197

198

\section{References}

Beyer M, Pogoda F, Pallez M, Lazic J, Hoffmann L, Pasquali M. 2014. Evidence for a reversible drought induced shift in the species composition of mycotoxin producing Fusarium head blight pathogens isolated from symptomatic wheat heads. International Journal of Food Microbiology 182-183:51-56.

Crespo-Sempere A, Estiarte N, Marín S, Sanchis V, Ramos AJ. 2015. Targeting Fusarium graminearum control via polyamine enzyme inhibitors and polyamine analogs. Food Microbiology 49:95-103.

De Kuppler ALM, Steiner U, Sulyok M, Krska R, Oerke E-C. 2011. Genotyping and phenotyping of Fusarium graminearum isolates from Germany related to their mycotoxin biosynthesis. International journal of food microbiology 151:78-86.

Desjardins AE. 2008. Natural Product Chemistry Meets Genetics: When Is a Genotype a Chemotype? Journal of Agricultural and Food Chemistry 56:7587-7592. 
199 Gardiner D, Kazan K, Praud S, Torney F, Rusu A, Manners J. 2010. Early activation of wheat

200 polyamine biosynthesis during Fusarium head blight implicates putrescine as an inducer of

201 trichothecene mycotoxin production. BMC Plant Biology 10:289.

202 Gardiner DM, Kazan K, Manners JM. 2009b. Nutrient profiling reveals potent inducers of

203 trichothecene biosynthesis in Fusarium graminearum. Fungal Genetics and Biology 46:604-613.

204 Gardiner DM, Osborne S, Kazan K, Manners JM. 2009. Low pH regulates the production of

205 deoxynivalenol by Fusarium graminearum. Microbiology: 155:3149-3156.

206 Giraud F, Pasquali M, Jarroudi ME, Vrancken C, Brochot C, Cocco E, Hoffmann L, Delfosse P,

207 Bohn T. 2010. Fusarium head blight and associated mycotoxin occurrence on winter wheat in

208 Luxembourg in 2007/2008. Food Additives \& Contaminants: Part A 27:825-835.

209 Hestbjerg H, Nielsen KF, Thrane U, Elmholt S. 2002. Production of trichothecenes and other

210 secondary metabolites by Fusarium culmorum and Fusarium equiseti on common laboratory

211 media and a soil organic matter agar: an ecological interpretation. Journal of Agricultural and

212 Food Chemistry 50:7593-7599.

213 Ji F, Wu J, Zhao H, Xu J, Shi J. 2015. Relationship of Deoxynivalenol Content in Grain, Chaff,

214 and Straw with Fusarium Head Blight Severity in Wheat Varieties with Various Levels of

215 Resistance. Toxins 7:728-742.

216 Jiao F, Kawakami A, Nakajima T. 2008. Effects of different carbon sources on trichothecene

217 production and Tri gene expression by Fusarium graminearum in liquid culture. FEMS

218 Microbiology Letters 285:212-219.

219 Kim H, Son H, Lee Y-W. 2014. Effects of light on secondary metabolism and fungal

220 development of Fusarium graminearum. Journal of Applied Microbiology 116:380-389. 
221 Kimura M, Tokai T, Takahashi-Ando N, Ohsato S, Fujimura M. 2007. Molecular and Genetic

222 Studies of Fusarium Trichothecene Biosynthesis: Pathways, Genes, and Evolution. Bioscience,

223 Biotechnology, and Biochemistry 71:2105-2123.

224 Llorens A, Mateo R, Hinojo MJ, Valle-Algarra FM, Jiménez M. 2004. Influence of

225 environmental factors on the biosynthesis of type B trichothecenes by isolates of Fusarium spp.

226 from Spanish crops. International Journal of Food Microbiology 94:43-54.

227 Magan N, Hope R, Colleate A, Baxter ES. 2002. Relationship Between Growth and Mycotoxin

228 Production by Fusarium species, Biocides and Environment. European Journal of Plant

229 Pathology 108:685-690.

230 Merhej J, Boutigny AL, Pinson-Gadais L, Richard-Forget F, Barreau C. 2010. Acidic pH as a

231 determinant of TRI gene expression and trichothecene B biosynthesis in Fusarium graminearum.

232 Food Additives \& Contaminants: Part A 27:710-717.

233 Newell SY, Statzell-Tallman A. 1982. Factors for Conversion of Fungal Biovolume Values to

234 Biomass, Carbon and Nitrogen: Variation with Mycelial Ages, Growth Conditions, and Strains 235 of Fungi from a Salt Marsh. Oikos 39:261-268.

236 Pasquali M, Beyer M, Bohn T, Hoffmann L. 2011. Comparative Analysis of Genetic

237 Chemotyping Methods for Fusarium: Tri13 Polymorphism Does not Discriminate between 3-

238 and 15-acetylated Deoxynivalenol Chemotypes in Fusarium graminearum. Journal of

239 Phytopathology 159:700-704.

240 Pasquali M, Migheli Q. 2014. Genetic approaches to chemotype determination in type B-

241 trichothecene producing Fusaria. International Journal of Food Microbiology 189:164-182. 
242 Pasquali M, Serchi T, Cocco E, Leclercq CC, Planchon S, Guignard C, Renaut J, Hoffmann L.

243 2015. A Fusarium graminearumstrain-comparative proteomic approach identifies regulatory

244 changes triggered by agmatine. Journal of Proteomics. 10.1016/j.jprot.2015.11.010

245 Piec J, Pallez M, Beyer M, Vogelgsang S, Hoffmann L, Pasquali M. Luxembourg database of

246 trichothecene type B Fusarium graminearum and F. culmorum producers. Bioinformation,

247 accepted for publication.

248 Pinson-Gadais L, Richard-Forget F, Frasse P, Barreau C, Cahagnier B, Richard-Molard D,

249 Bakan B. 2008. Magnesium represses trichothecene biosynthesis and modulates Tri5, Tri6, and

250 Tri12 genes expression in Fusarium graminearum. Mycopathologia 165:51-59.

251 Ponts N, Couedelo L, Pinson-Gadais L, Verdal-Bonnin M-N, Barreau C, Richard-Forget F.

252 2009. Fusarium response to oxidative stress by H2O2 is trichothecene chemotype-dependent.

253 FEMS microbiology letters 293:255-262.

254 Ponts N, Pinson-Gadais L, Barreau C, Richard-Forget F, Ouellet T. 2007. Exogenous H2O2 and

255 catalase treatments interfere with Tri genes expression in liquid cultures of Fusarium

256 graminearum. FEBS Letters 581:443-447.

257 Proctor RH, McCormick SP, Alexander NJ, Desjardins AE. 2009. Evidence that a secondary

258 metabolic biosynthetic gene cluster has grown by gene relocation during evolution of the

259 filamentous fungus Fusarium. Molecular Microbiology 74:1128-1142.

260 Schmidt-Heydt M, Parra R, Geisen R, Magan N. 2011. Modelling the relationship between

261 environmental factors, transcriptional genes and deoxynivalenol mycotoxin production by strains

262 of two Fusarium species. Journal of The Royal Society Interface 8:117-126. 
263 Sieber CMK, Lee W, Wong P, Münsterkötter M, Mewes H-W, Schmeitzl C, Varga E, Berthiller

264 F, Adam G, Güldener U. 2014. The Fusarium graminearum Genome Reveals More Secondary

265 Metabolite Gene Clusters and Hints of Horizontal Gene Transfer. PLoS ONE 9:e110311.

266 Tsuyuki R, Yoshinari T, Sakamoto N, Nagasawa H, Sakuda S. 2011. Enhancement of

267 trichothecene production in Fusarium graminearum by cobalt chloride. Journal of agricultural 268 and food chemistry 59:1760-1766.

269 Valdes-Santiago L, Cervantes-Chavez JA, Leon-Ramirez CG, Ruiz-Herrera J. 2012. Polyamine 270 metabolism in fungi with emphasis on phytopathogenic species. Journal of Amino Acids;

271 e837932.

272 von der Ohe C, Gauthier V, Tamburic-Ilincic L, Brule-Babel A, Fernando W, Clear RM, Ward 273 T, Miedaner T. 2011. A comparison of aggressiveness and deoxynivalenol production between

274 Canadian Fusarium graminearum isolates with 3-acetyl and 15-acetyldeoxynivalenol

275 chemotypes in field-grown spring wheat. European Journal of Plant Pathology 127: 407-417.

276 Waśkiewicz A, Morkunas I, Bednarski W, Mai VC, Formela M, Beszterda M, Wiśniewska H, 277 Goliński P. 2014. Deoxynivalenol and Oxidative Stress Indicators in Winter Wheat Inoculated 278 with Fusarium graminearum. Toxins 6:575-591. 
283 Mass in mg of dried mycelium grown 10 days in the two media (Gardiner medium

284 containing agmatine; Jiao medium containing glutamic acid). Asterisks indicate significant

285 differences using the Mann-Whitney rank test $(\mathrm{p}<0.05)$.

\section{Figure 2. Toxin ratio comparing the two media.}

287 Log 10 transformed the average ratio of TB production in the agmatine vs the glutamic 288 acid medium. Values are the sum of all trichothecene type B detected (DON, 3ADON, 289 15ADON, NIV, FUSX). Isolates that have a higher amount of TB in the glutamic acid 290 medium are in red while those that have a higher amount in the agmatine medium are in 291 blue 


\section{Figure 1 (on next page)}

Dry mycelium mass measured in the two media in the three species.

Mass in $\mathrm{mg}$ of dried mycelium grown 10 days in the two media (Gardiner medium containing agmatine; Jiao medium containing glutamic acid). Asterisks indicate significant differences using Mann-Whitney rank test $(p<0.05)$. 


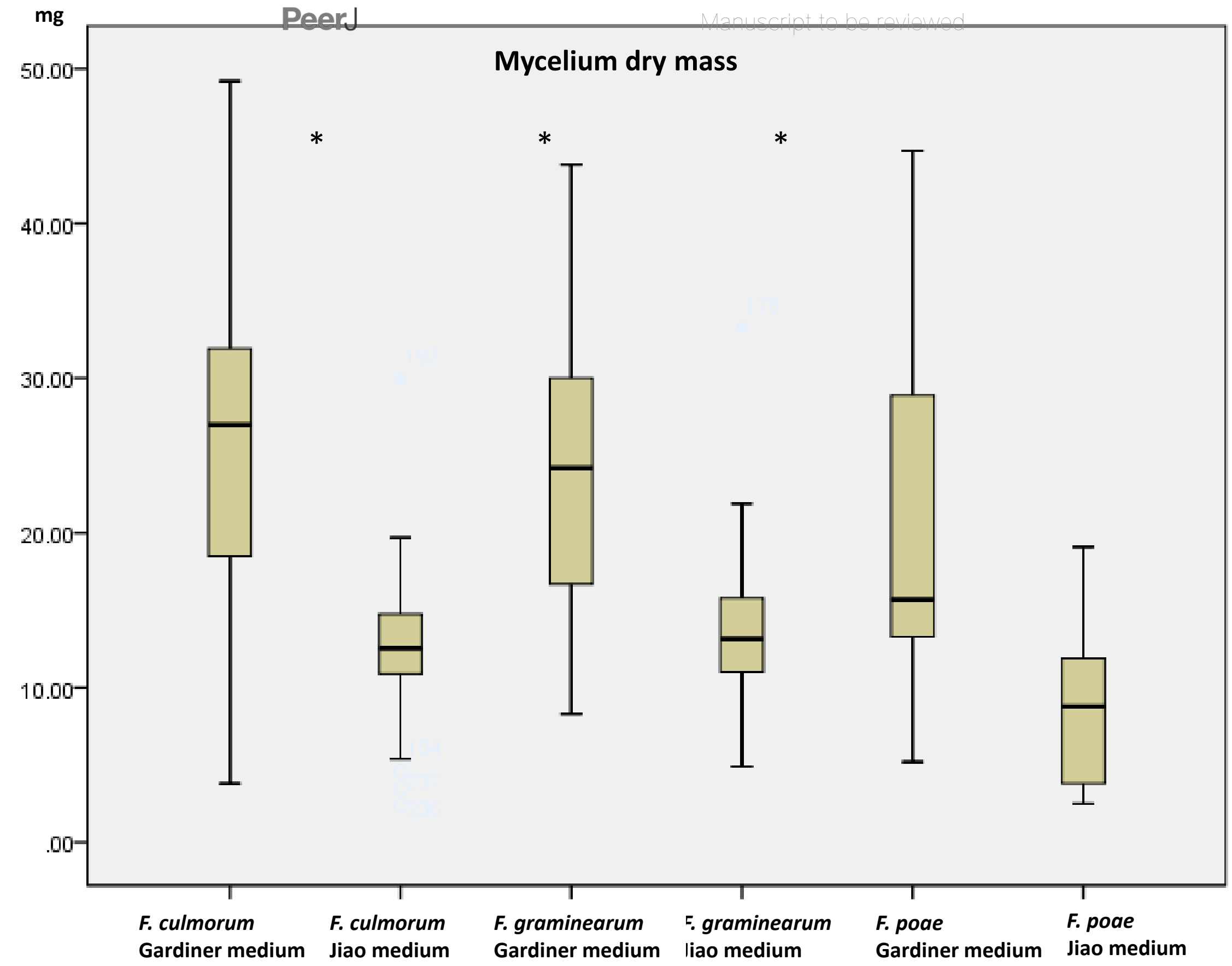




\section{Figure 2 (on next page)}

Toxins ratio comparing the two media.

Log 10 transformed average ratio of TB production in agmatine vs glutamic acid medium. Values are the sum of all trichothecene type B detected (DON, 3ADON, 15ADON, NIV, FUSX). In red, isolates that have higher amount of TB in the glutamic acid medium while in blue those that have higher amount in the agmatine medium. 


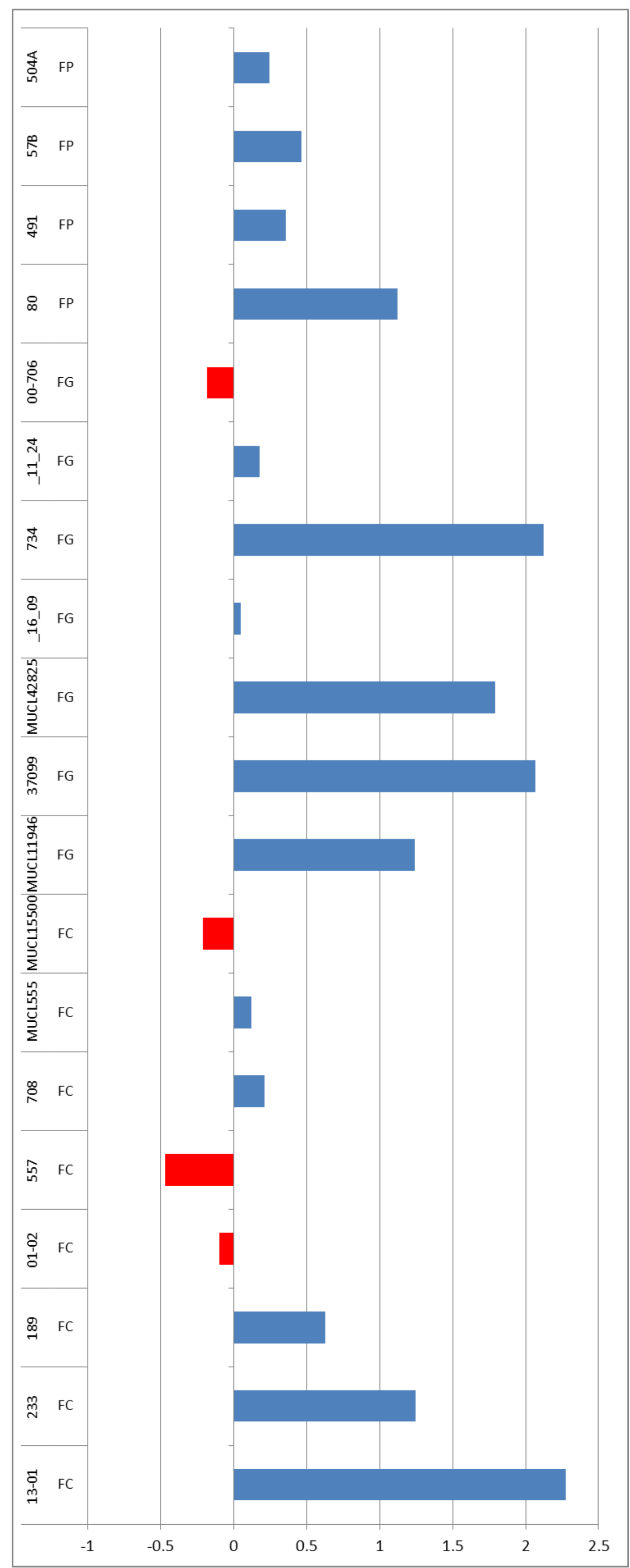

PeerJ reviewing PDF | (2015:06:5410:2:0:NEW 18 Jan 2016) 


\section{Table $\mathbf{1}$ (on next page)}

Table 1

Strain identification code, species, genetic chemotype, year of isolation, geographical origin, strain collection where the strain is deposited. 


\begin{tabular}{|c|c|c|c|c|c|}
\hline $\begin{array}{c}\text { Strain } \\
\text { identification } \\
\text { code }\end{array}$ & Species & $\begin{array}{c}\text { Genetic } \\
\text { chemotype }\end{array}$ & $\begin{array}{c}\text { Year of } \\
\text { isolation }\end{array}$ & $\begin{array}{c}\text { Geographical } \\
\text { origin }\end{array}$ & $\begin{array}{c}\text { Strain } \\
\text { collection* }\end{array}$ \\
\hline $13-01$ & F. culmorum & $3 \mathrm{ADON}$ & 2008 & $\begin{array}{l}\text { (Hoscheid) } \\
\text { Luxembourg } \\
\text { (Reisdorf) }\end{array}$ & LuxMCC \\
\hline 233 & F. culmorum & NIV & 2007 & Luxembourg & LuxMCC \\
\hline 189 & F. culmorum & $3 \mathrm{ADON}$ & 2007 & $\begin{array}{c}\text { (Reisdorf) } \\
\text { Luxembourg }\end{array}$ & LuxMCC \\
\hline $01-02$ & F. culmorum & NIV & 2008 & $\begin{array}{c}\text { (Kehlen) } \\
\text { Luxembourg }\end{array}$ & LuxMCC \\
\hline 557 & F. culmorum & $3 \mathrm{ADON}$ & 2007 & $\begin{array}{c}\text { (Reuler) } \\
\text { Luxembourg }\end{array}$ & LuxMCC \\
\hline 708 & F. culmorum & NIV & 2007 & $\begin{array}{l}\text { (Christnach) } \\
\text { Luxembourg }\end{array}$ & LuxMCC \\
\hline MUCL555 & F. culmorum & NIV & 1952 & Unknown & MUCL \\
\hline MUCL15500 & F. culmorum & NIV & 1946 & Netherlands & MUCL \\
\hline MUCL 11946 & $F$. graminearum & $3 \mathrm{ADON}$ & 1969 & Belgium & MUCL \\
\hline NRRL37099 & $F$. graminearum & $3 \mathrm{ADON}$ & 1994 & $\begin{array}{c}\text { (Manitoba) } \\
\text { Canada }\end{array}$ & NRRL \\
\hline MUCL 42825 & $F$. graminearum & NIV & 2000 & Belgium & MUCL \\
\hline $16-09$ & F. graminearum & NIV & 2008 & $\begin{array}{l}\text { (Troisvierges) } \\
\text { Luxembourg }\end{array}$ & LuxMCC \\
\hline 734 & F. graminearum & $15 \mathrm{ADON}$ & 2007 & $\begin{array}{l}\text { (Christnach) } \\
\text { Luxembourg }\end{array}$ & LuxMCC \\
\hline $11-24$ & F. graminearum & $15 \mathrm{ADON}$ & 2008 & $\begin{array}{l}\text { (Echternach) } \\
\text { Luxembourg }\end{array}$ & LuxMCC \\
\hline UMW00-706 & $F$. graminearum & $15 \mathrm{ADON}$ & $>2000$ & USA & $\begin{array}{c}\text { Courtesy of } \\
\text { L. Gale }\end{array}$ \\
\hline 80 & $F$. poae & - & 2007 & $\begin{array}{l}\text { (Nothum) } \\
\text { Luxembourg }\end{array}$ & LuxMCC \\
\hline 491 & $F$. poae & - & 2007 & $\begin{array}{l}\text { (Nothum) } \\
\text { Luxembourg }\end{array}$ & LuxMCC \\
\hline $57 \mathrm{~B}$ & $F$. poae & - & 2007 & $\begin{array}{c}\text { (Kayl) } \\
\text { Luxembourg }\end{array}$ & LuxMCC \\
\hline $504 \mathrm{~A}$ & $F$. poae & - & 2007 & $\begin{array}{c}\text { (Kayl) } \\
\text { Luxembourg }\end{array}$ & LuxMCC \\
\hline
\end{tabular}

Table 1. Strain identification code, species, genetic chemotype, year of isolation, geographical origin, strain collection where the strain is deposited. de Louvain (BEL); NRRL: Agricultural Research Service culture collection (USA). 